\title{
Frakturendoprothetik bei Schenkelhalsfrakturen
}

\author{
Philipp Bula, Felix Bonnaire
}

\section{Zusammenfassung}

Weltweit hat die Zahl der Hüftendoprothesenimplantationen in den letzten Jahren stetig zugenommen. Von den 200000 in Deutschland implantierten Hüftendoprothesen erfolgte bereits jede 4 . Versorgung aufgrund einer Fraktur. Aktuell ist die endoprothetische Versorgung die häufigste Therapie der Schenkelhalsfraktur in Deutschland [1] mit steigendem Anteil, obwohl es bislang keinen nachgewiesenen Vorteil der TEP-Versorgung gegenüber der Osteosynthese in Hinblick auf das Outcome gibt [2]. Die Frage, mit welcher Art von Prothese die Frakturversorgung erfolgen soll, hängt von zahlreichen Faktoren ab und sollte das numerische wie das biologische Alter des Patienten, Dislokationsgrad und Frakturmorphologie sowie Komorbiditäten und funktionellen Anspruch mit einbeziehen. Bei Patienten bis zum 60. Lebensjahr sollte in der Behandlung unabhängig vom Dislokationsgrad der Fraktur immer auf einen Gelenkerhalt abgezielt werden (pathologische Frakturen ausgeschlossen). Aber auch die nicht dislozierten und valgisch impaktierten Schenkelhalsfrakturen können in allen Altersklassen mit Vorteil gegenüber der Prothese gelenkerhaltend operiert werden. Bei dislozierten Frakturen profitieren ältere und körperlich reduzierte Patienten (>65 Jahre) von einer Totalendoprothese. Umso mehr bei begleitend bestehender Koxarthrose. Hemiprothesen sind in aller Regel den alten multimorbiden Patienten vorbehalten, die hier v.a. von der kürzeren OP-Zeit und dem geringeren Blutverlust profitieren. Grundsätzlich wird ab dem 70. Lebensjahr die Verwendung zementierter Prothesen empfohlen [3]. Bei der Frakturendoprothetik handelt es sich, ebenso wie bei der Osteosynthese, um eine Operation von hoher Dringlichkeit, die zur Reduktion der Mortalität und sekundärer Komplikationen innerhalb von maximal 48 Stunden erfolgen muss [4-6]. Dieser Umstand verdeutlicht, dass der Operateur einer Schenkelhalsfraktur sowohl die Endoprothetik wie auch die Osteosynthese beherrschen sollte.

\section{Fracture Endoprosthesis in Femoral Neck Fractures}

The number of hip arthroplasties is increasing throughout the world. Of the total of 200,000 implanted hip endoprostheses in Germany, 25\% are due to a femoral neck fracture. This means that hip replacement is now the most common procedure for surgical treatment in femoral neck fractures in Germany [1]. This is tending to increase, although there is no evidence that the outcome is better with hip replacement than with osteosynthesis [2]. The choice of the implant depends on many factors, including the patient's calendar and biological ages, the fracture type and degree of dislocation as well as functional demands and comorbidities. In younger patients (up to 60 years), therapy should be focussed on joint preservation, aside from the degree of dislocation. Non-displaced and valgus impacted femoral neck fractures can be treated to preserve the joint with ostheosynthesis in all age groups and is superior to hip replacement. In displaced fractures, elder and physically unfit patients ( $>60$ years) benefit from total hip replacement, particularly with preexisting coxarthrosis. Hemiprosthesis is usually reserved for older and multimorbid patients, who benefit from the shorter operation time and lower blood loss in this procedure. Cemented prostheses are generally recommended for patients older than 70 years [3]. Like osteosynthesis, endoprosthetic fracture care is a surgical procedure of high priority that must take place within a maximum of 48 hours, in order to reduce mortality and secondary complications [4-6]. This makes it clear that the surgeon who is treating femoral neck fractures should master both procedures - hip replacement and osteosynthesis.

\section{Einleitung}

Schenkelhalsfrakturen sind mittlerweile neben den distalen Radiusfrakturen, den Oberarmkopffrakturen und Knöchelfrakturen die häufigsten Frakturen des Erwachsenenalters (etwa 60000 pro

OP-JOURNAL 2016; 32: 206-214

(c) Georg Thieme Verlag KG Stuttgart · New York DOI http://dx.doi.org/10.1055/s-0036-1595902
Jahr in Deutschland 2014). Die Inzidenz für die Gesamtbevölkerung liegt bei etwa 90/100000 und nimmt ab dem 65. Lebensjahr nahezu exponentiell auf knapp 1000/100000 zu [7,8]. Zwei Drittel dieser Frakturen betreffen Frauen um das 80. Lebensjahr und in nahezu allen Fällen handelt es sich um osteoporoseassoziierte Frakturen [9]. Schenkelhalsfrakturen werden heute grundsätzlich operativ versorgt. Es gibt nur noch wenige Ausnahmeindikationen für die kon- servativ-funktionelle Behandlung, die entsprechend auch in den Leitlinien der DGU von 2015 nicht mehr favorisiert wird. Die operative Behandlung sollte auf die Bedürfnisse des jeweiligen Patienten eingehen und sowohl die Prognose der Frakturbehandlung, Komplikationsmöglichkeiten als auch die individuell unterschiedlichen Ausgangslagen (Belastung und Anspruch) mit einbeziehen. Im Großen und Ganzen werden die Frakturen des jungen Menschen mit gro- 


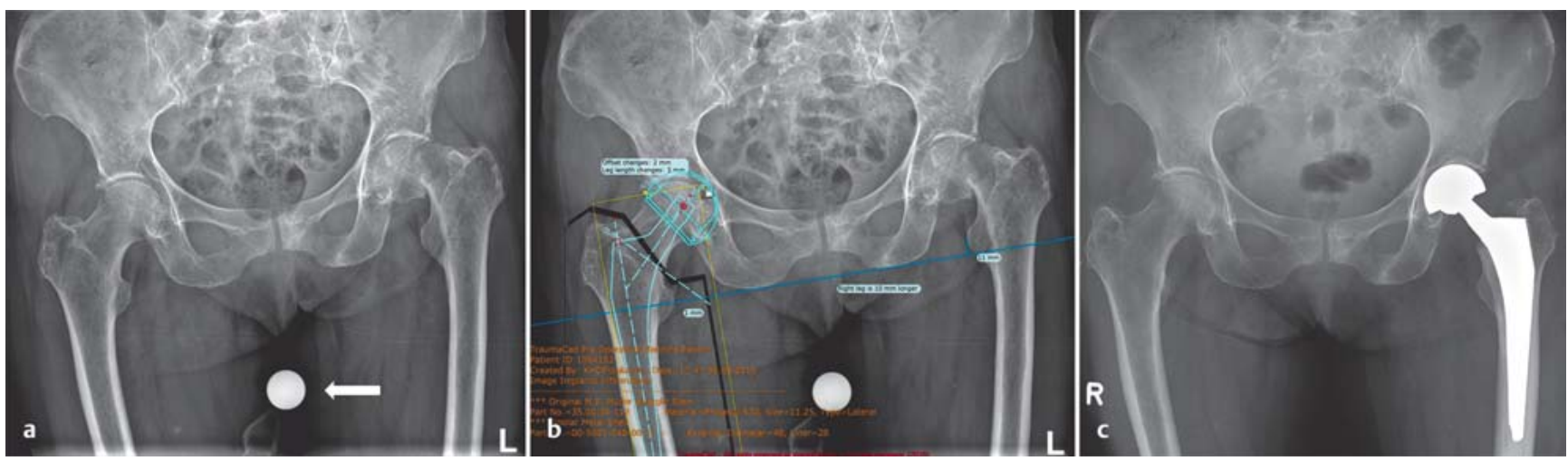

Abb. 1 a bis c 89-jährige Patientin mit Garden-IV-Fraktur. Unfallsituation mit tief eingestellter Beckenübersicht und Kalibrierungs-Tool für die maßstabsgerechte Planung (a). Abbildung b zeigt die präoperative Planung an der unverletzten Gegenseite. Abbildung c zeigt die implantierte bipolare Hemiprothese (Duokopf).

ßen Belastungen und hohem Anspruch von denen der älteren Patienten mit eingeschränkter Mobilität und geringerem funktionellem Anspruch unterschieden. Dazwischen findet sich eine große Grauzone, die einer exakten Bewertung der Begleitumstände und individuellen ärztlichen Beratung des Patienten bedarf.

Aufgrund der alternden Bevölkerung mit großer Zahl an multimorbiden Patienten ist die 1-Jahres-Mortalität anhaltend groß und liegt nach wie vor bei etwa $25 \%[4,10,11]$.

Aufgrund der hohen Lockerungsraten bei jungen Patienten werden Endoprothesen bei dieser Patientengruppe so weit wie möglich vermieden. Je jünger die Patienten, je höher die Beanspruchung und die Lebensbelastungszeit der Hüfte, desto häufiger sind auch Lockerungen der Endoprothese zu erwarten. Diese führen zu zunehmendem Knochenverlust und Wechseloperationen mit zwangsläufig auch zunehmenden Komplikationen. In der Gruppe der Menschen bis 60 Jahre sollte deswegen alles versucht werden, um das Hüftgelenk zu erhalten. Für diese Patienten ist grundsätzlich die Osteosynthese primäres Ziel der Behandlungsoptionen.

Die Patienten ab 60 Jahren profitieren bei dislozierten Frakturen von einer individuell angepassten Endoprothetik (vollständig zementfrei, Hybrid oder voll zementiert, Hemiprothese oder TEP).

Zwischen 60 und 70 Jahren liegen viele individuelle Konstellationen, bei denen der Einsatz des einen oder anderen Verfahrens sinnvoll ist. Biologisch jüngere Patienten mit dislozierten Frakturen profitieren mehr von zementfreien, knochensparenden Prothesenmodellen, äl- tere Patienten haben Vorteile von Hybridvarianten und ab 70 Jahren werden eher zementierte Prothesen für Pfanne und Schaft empfohlen [3].

Die alten und multimorbiden Patienten mit geringem Anspruch und eingeschränkter Lebenserwartung profitieren hingegen eher von einer zementierten Hemiprothese, deren Implantation aufgrund der kürzeren OP-Zeit und des geringeren Blutverlusts weniger belastend ist. Auch die Luxationsrate der Hemiprothesen ist gegenüber der TEP signifikant geringer [12]. In Deutschland werden heute fast ausschließlich bipolare Modelle verwendet, die gegenüber den früher gängigen unipolaren Prothesen eine bessere Funktion und weniger Schmerzen erreichen [13].

\section{Therapieziel}

Für die Frakturendoprothetik ist grundsätzlich eine möglichst komplikationsarme Implantation einer für den Patienten nach seinen Ansprüchen ausgerichteten Implantatkombination mit möglichst lebenslanger Haltbarkeit auszuwählen. Die durchgeführte Operation muss eine sofortige postoperative Vollbelastung ermöglichen, um die Grundvoraussetzung für ein rasches Wiedererlangen des gewohnten Aktivitätslevels zu schaffen.

\section{Diagnostik}

In der Regel wird bei dislozierten Frakturen die Diagnose durch die Verkürzung und Außenrotation des betroffenen Beines einfach gestellt. Dann kommt nur noch eine Schenkelhals- oder eine pertrochantäre Fraktur infrage.

Für die Planung der Versorgung einer Schenkelhalsfraktur ist eine Beckenüber- sicht mit Kalibrierungs-Tool notwendig (Abb. 1). Patienten mit starken Schmerzen ohne Frakturnachweis im konventionellen Röntgen sollten eine CT-Untersuchung von Becken und Hüften erhalten, um nicht dislozierte hüftnahe Frakturen und Frakturen am hinteren Beckenring zu identifizieren (Abb.2). Die CT-basierte 3-D-Darstellung der Hüftknochen ist hilfreich für die Einschätzung der Prognose, der Operationsmethode und vor allem für die Reposition von Frakturen (Abb. 3).

Entscheidend für die Wahl des Vorgehens ist neben der Frakturform die exakte Anamnese mit Erfassung der Begleitumstände des Patienten: Macht er Sport und welchen, ist er voll selbstständig, braucht er Gehhilfen oder wohnt er im Heim, und wie ist der allgemeine körperliche Zustand, gibt es Risikofaktoren für Embolien, nimmt er Antikoagulanzien ein, hat er Herz-Gefäß- oder Lungenerkrankungen usw.?

\section{Entscheidungsfindung}

Die Entscheidung für das richtige operative Verfahren ist die schwierigste Aufgabe.

Die Therapiewahl hängt von vielen Voraussetzungen ab. In der Reihenfolge der Wichtung ist das Dislokationsausmaß führend: Nicht dislozierte und valgisch impaktierte Schenkelhalsfrakturen können in allen Altersklassen mit Vorteilen gegenüber der Prothese gelenkerhaltend operiert werden. Lokaler Tumorbefall ausgeschlossen. Für die jüngeren Patienten steht die dynamische Hüftschraube (DHS) oder alternativ eine Schraubenosteosynthese zur Verfügung. Letztere erlaubt allerdings in aller Regel keine postoperative Vollbelastung und 


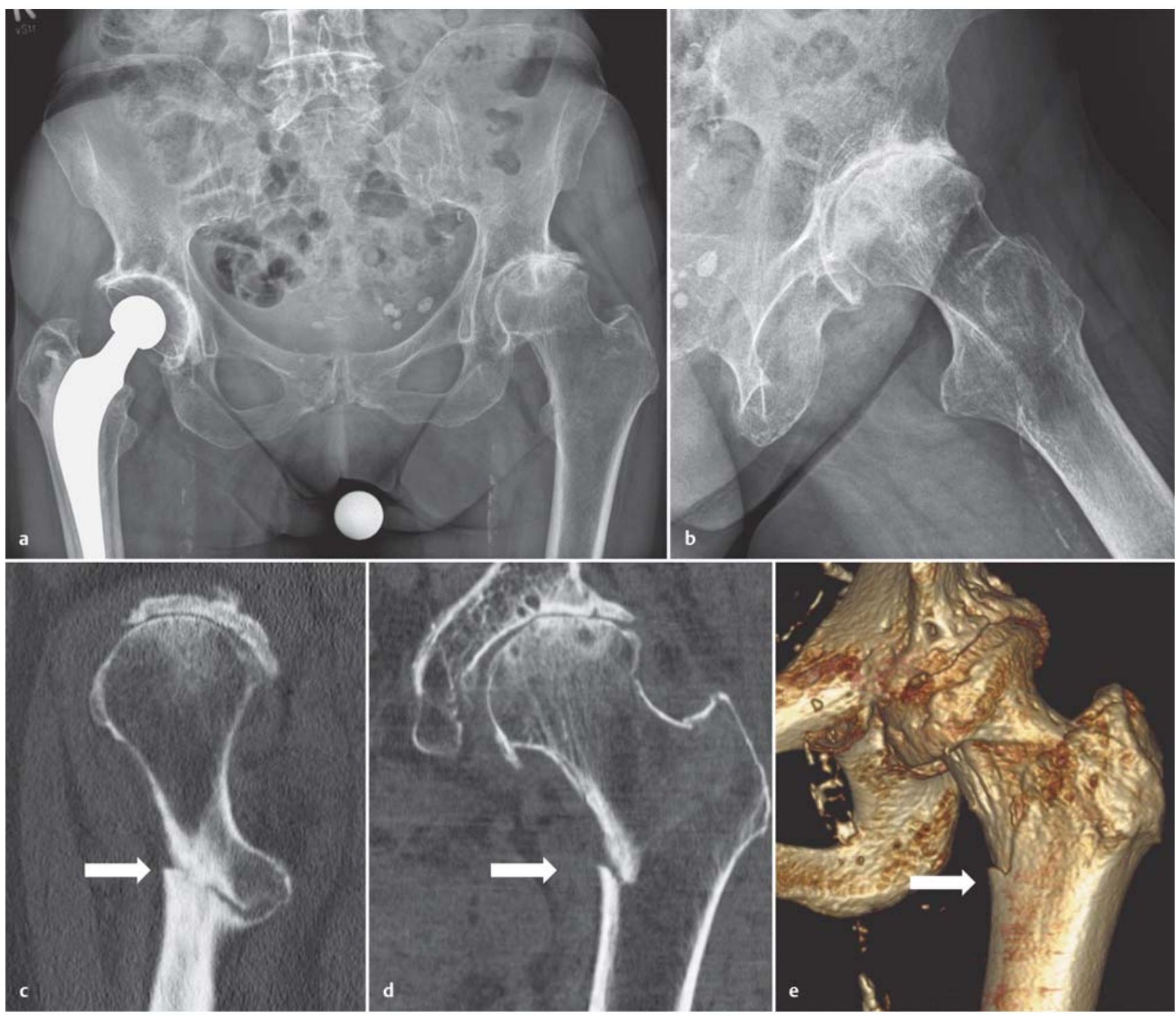

Abb. 2 a bis e 84-jährige Patientin nach Sturz auf die linke Hüfte. Anhaltend geh- und stehunfähig aufgrund von Schmerzen. Konventionell-radiologisch kein Frakturnachweis (a und b). Die CT zeigt die A1-Fraktur (c bis e).

ist der DHS damit unterlegen. Bei reduzierter Knochenqualität und absehbar nicht notwendiger Materialentfernung empfiehlt sich für die Älteren eher das stabilere Implantat mit Klinge (DHS Blade, Synthes) oder alternativ mit mehreren Schrauben (z.B. Targon FN, Braun).

Bei dislozierten Frakturen ist das Alter entscheidend, dann das Aktivitätslevel (sportliche, private und beufsbezogene Ansprüche), Nebenerkrankungen wie Alkoholkrankheit, Niereninsuffizienz, kardiopulmonale Erkrankungen, Osteoporose, Demenz oder neurologische Erkrankungen und die Lebensumstände (in Familie, allein lebend, aber selbstständig, in Pflegeeinrichtungen, Rehapotenzial).

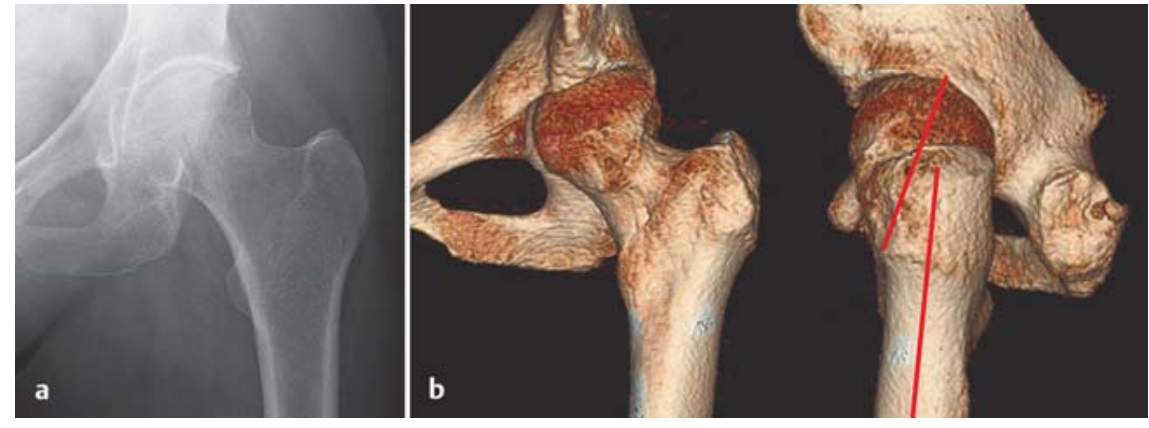

Abb. $\mathbf{3}$ a und $\mathbf{b}$ 75-jährige Patientin mit Insuffizienzfraktur des Schenkelhalses. Die exakte Dislokation ist im konventionellen Röntgenbild nicht ausreichend erfasst (a). CT zur Planung der Operation und Reposition (b).

Junge, gesunde Patienten sollten immer gelenkerhaltend operiert werden. Eine feste Grenze gibt es nicht, in manchen Ländern liegt sie bei 60 Jahren, in anderen bei 75 Jahren. Bei der Entscheidung helfen die Frakturmorphologie und die Nebenerkrankungen.

Gering verschobene Frakturen mit relativ großem Kopffragment lassen sich gut 


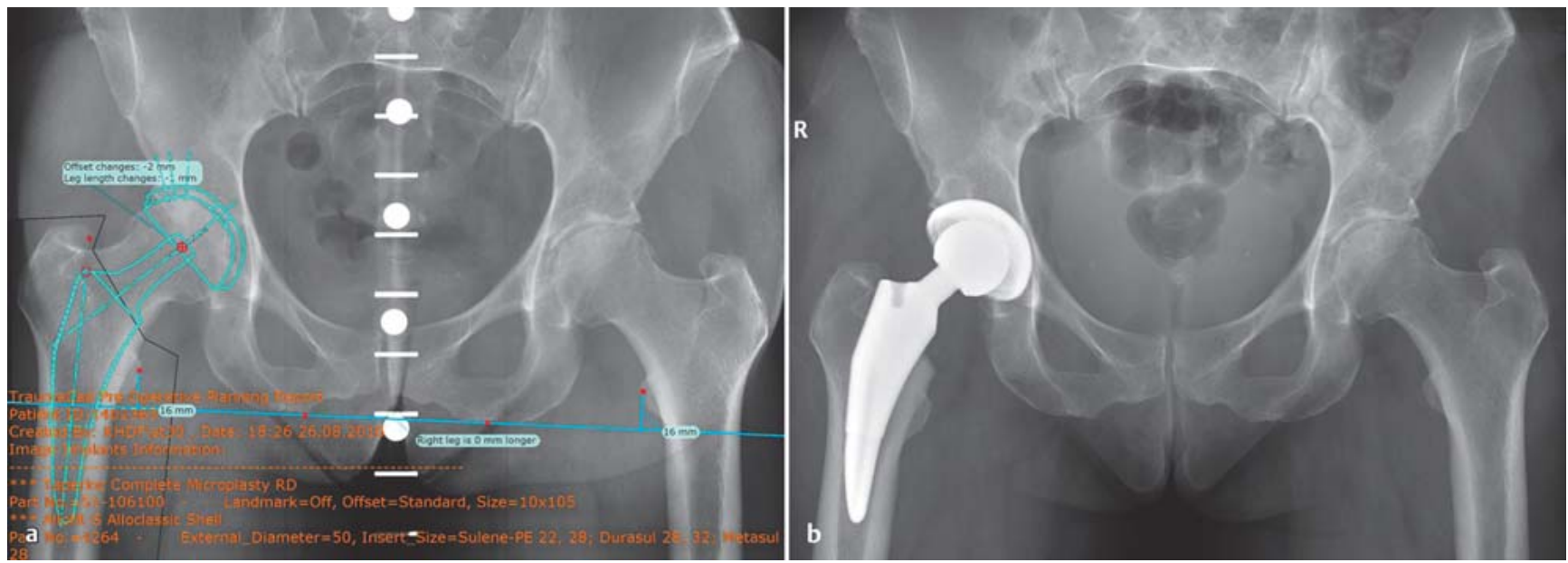

Abb. 4a und b Digitale Planung der funktionellen Anatomie mit Beinlängendifferenz und Ausführung: Digitale Planung der Implantatpositionierung und -größe (a) sowie die postoperative Röntgenkontrolle (b).

fixieren. Verschobene Frakturen mit schräg verlaufenden Brüchen in 2 Ebenen und kleinem Kopffragment lassen sich nicht gut reponieren und stabilisieren. Trümmerzonen haben eine höhere Pseudarthoserate und neigen zur Schenkelhalsverkürzung [14]. Garden-IV-Verletzungen sind grundsätzlich schwer zu reponieren und haben ein hohes Risiko für die Entstehung einer Femurkopfnekrose.

Je mehr Nebenerkrankungen, desto mehr spricht bei dislozierten Frakturen für die Prothese. Postoperative Vollbelastung ist in jedem Fall zu fordern.

Lässt sich ein Hüftgelenk bei biologisch jungen Patienten über 60 Jahren nicht mehr erhalten, werden heute knochensparende Operationen mit Kurzschaftprothesen bevorzugt. Sie werden als zementfreie Totalendoprothesen eingesetzt. Die bevorzugte Art der Pfanne ist eine Press-fit-Pfanne. Die Gleitpaarungen der Oberflächen von Kopf und Pfanne werden in der Literatur unterschiedlich bewertet. Metall auf ultragehärtetem Polyethylen scheint in dieser Gruppe eine sichere Wahl zu sein (Abb.4).

Im intermediären Anspruchsbereich können Hybridvarianten mit Press-fitPfannen und zementierten Schäften benutzt werden. Diese haben den Vorteil, dass der Schaft bei nachlassender Knochendichte im Zementköcher guten Halt findet. Bevorzugt wird ein Geradschaftmodell verwendet, was sich über Jahre bewährt hat und reproduzierbar hervorragende 10-Jahres-Standzeiten erwarten lässt [15].
Patienten ab 70 Jahren profitieren von zementierten Prothesen, sie haben nach der Operation weniger Schmerzen, eine bessere Funktion weniger Revisionsoperationen und meist bessere Langzeitergebnisse [3] (Abb.5).

Patienten mit Hüftdysplasien oder Luxationsneigung profitieren von tripolaren Prothesen, die Luxationen weitgehend verhindern (Abb. 6).

Für ältere Patienten, die nur noch wenige Ansprüche an die Mobilität und Beweglichkeit haben, setzt man heute bei dislozierten Frakturen die Duokopfprothese ein. Sie verfügt über 2 Rotationszentren in der Pfanne. Als Schaft wird i.d.R. ein zementierter Geradschaft ohne Kragen eingebracht. Die Duokopfprothese ist der kleinere, weniger belastende Eingriff. Die Frühergebnisse sind gut und die Luxationsrate deutlich geringer als bei den Totalendoprothesen [16] (Abb. 1).

\section{Klassifikationen}

Die Einteilung nach Pauwels (Abb.7a) hilft nur bei den eingestauchten Frakturen: diese sind i.d.R. stabil und sollten nur bei den jungen Patienten reponiert werden (CT sehr hilfreich). Bei älteren Patienten ist eine sekundäre Arthrose nicht zu erwarten. Die Garden-Klassifikation (Abb. 7b) ist ebenfalls nicht sehr verlässlich, sie hilft aber doch: Garden-Iund -II-Brüche können mittels Osteosynthese versorgt werden, bei III und IV wird es kritisch. Besser ist die 3-D-Darstellung im CT zur Beurteilung der Osteosynthesemöglichkeiten. Die AO-Klassifikation trennt im Wesentlichen die impaktierten Frakturen von denen mit großem Kopf-
Hals-Fragment und den subkapitalen Frakturen und berücksichtigt damit die Aspekte Perfusionsstörung, Stabilität und Fragmentdislokation (Abb. 7 c).

Alle 3 Klassifikationen für sich erfassen allerdings nicht die Dreidimensionalität der Verletzung und sind damit für sich allein nicht therapierelevant. Die 3 genannten Klassifikationen haben obendrein eine hohe Inter- und Intraobserver-Abweichung.

\section{Operatives Vorgehen und Versor- gungszeitpunkt}

Die operative Versorgung hüftnaher Frakturen ist ein Eingriff von hoher Dringlichkeit und sollte nach den Forderungen des Gemeinsamen Bundesausschusses innerhalb von 48 Stunden durchgeführt werden, Osteosynthesen sogar innerhalb von 24 Stunden. Es ist wissenschaftlich belegt, dass die frühzeitige Versorgung sowohl das Outcome verbessert als auch die Mortalität reduziert. Dies gilt für die Osteosynthese, aber auch für die endoprothetische Versorgung $[4-6,17]$. Bislang fehlt der wissenschaftliche Nachweis, dass eine Endoprothese der Osteosynthese in Bezug auf das Outcome bei medialer Schenkelhalsfraktur überlegen ist [2]. Unstrittig ist allerdings die höhere Reoperationsrate bei der Osteosynthese [18], vor allem der dislozierten Frakturen, was gerade für die älteren Patienten eine große Rolle spielt, da Reeingriffe diese Patientengruppe überproportional belasten.

Die frakturassoziierte Implantation einer Hüftendoprothese stellt somit eine klare Notfallindikation dar. Damit verbunden 


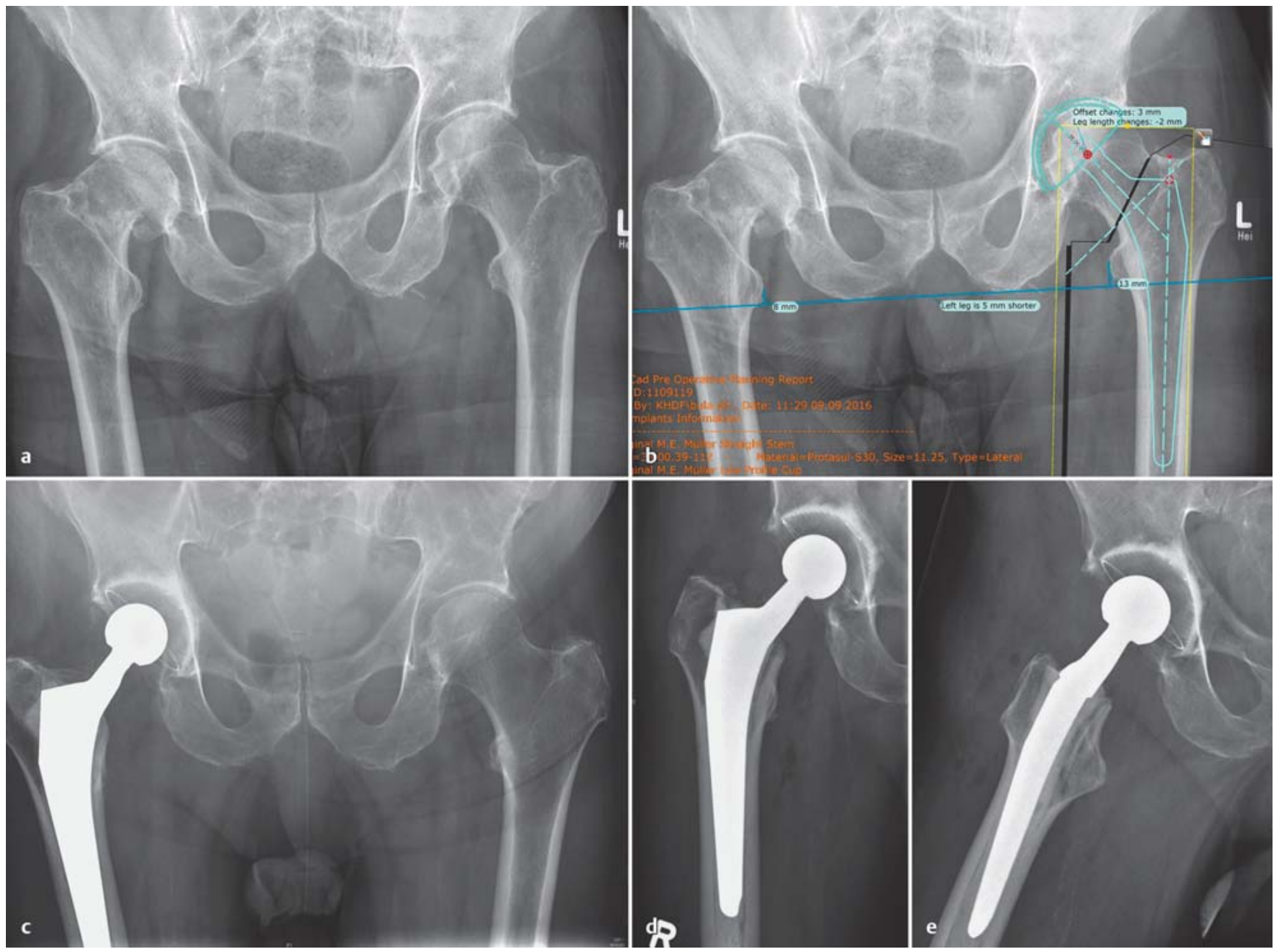

Abb. $\mathbf{5}$ a bis e 77-jähriger Patient, Sturz in der Straßenbahn, Nebendiagnose arterieller Hypertonus, körperlich sehr aktiv. Abb. a zeigt die Unfallbilder mit varisch dislozierter medialer Schenkelhalsfraktur Typ IV nach Garden. Planung der Prothese an der gesunden Gegenseite (b). Postoperative Situation (c-e).

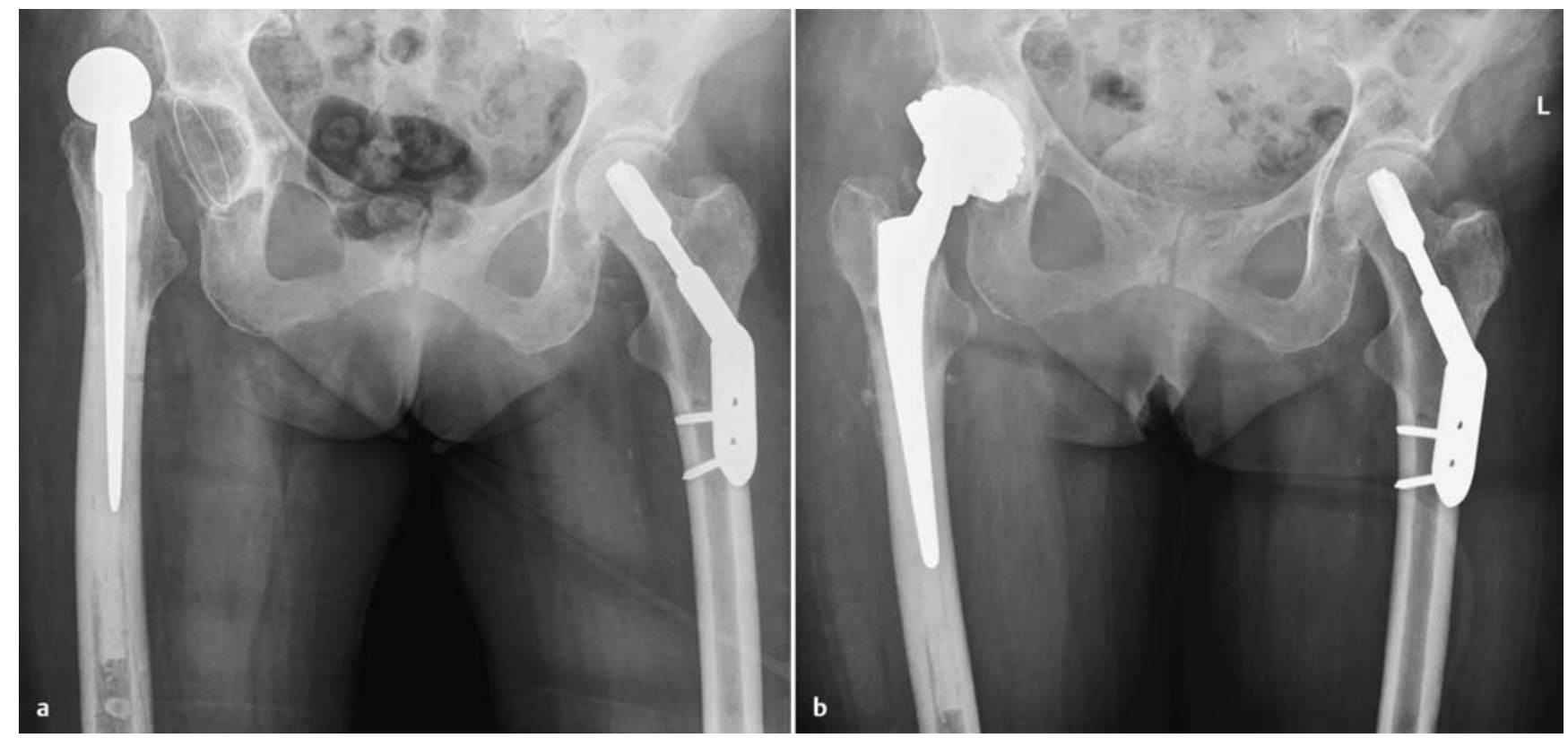

Abb. 6 a bis c 78-jährige Patientin mit Hüft-TEP rechts aufgrund Pseudarthrose einer Schenkelhalsosteosynthese mit rezidivierenden Prothesenluxationen bei fehlender muskulärer Abstützung und zu steil implantierter Pfanne. Wechsel auf tripolare Kopf-Pfannen-Kombination. 


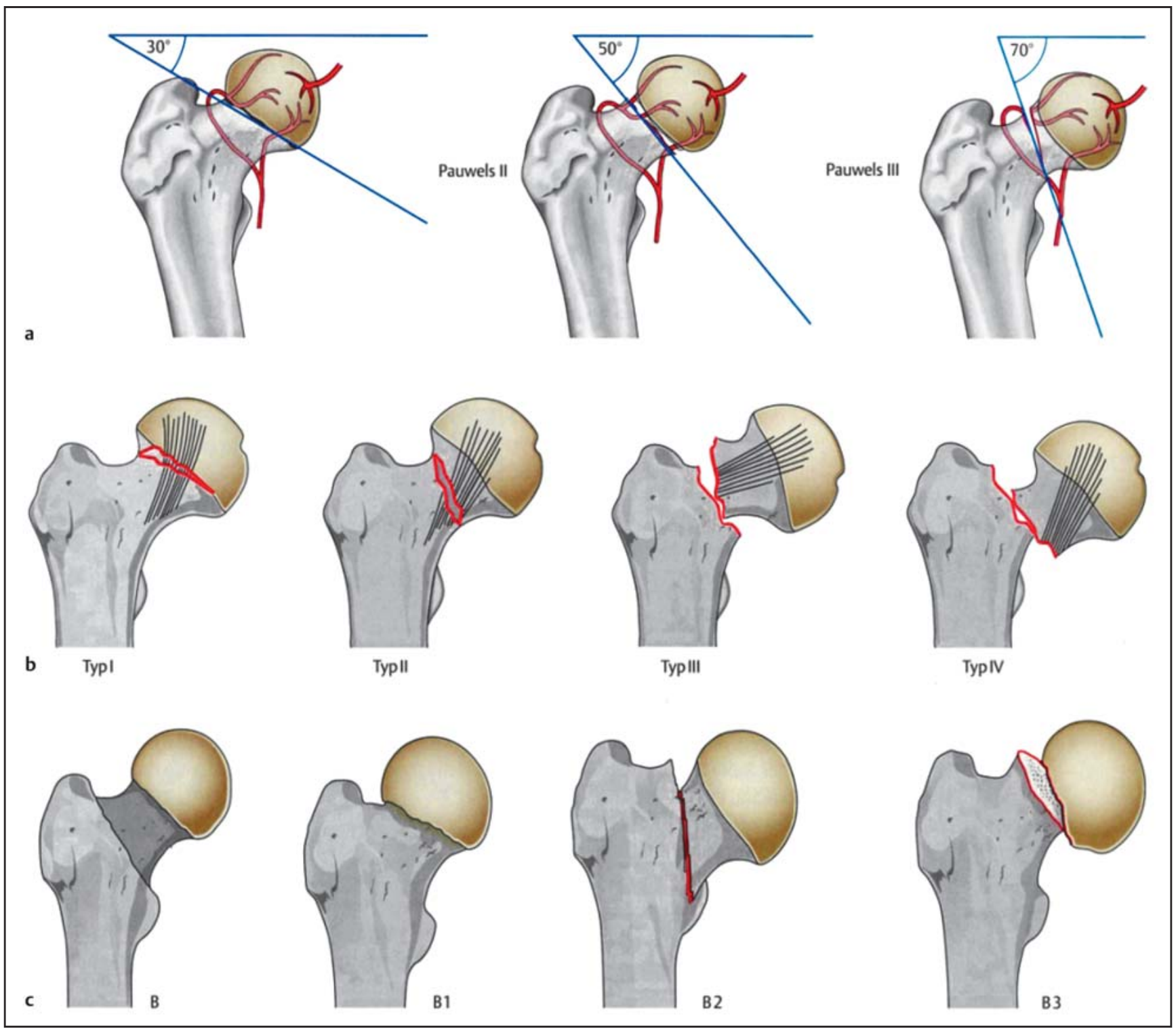

Abb. 7a bis c Klassifikationen Schenkelhalsfraktur nach Pauwels (a), Garden (b) und AO (c) (Bild: Bonnaire F et al. Hüftgelenknahe Frakturen/ Luxationen. Orthopädie und Unfallchirurgie up2date 2008; 3: 395-412).

ist natürlich auch eine deutlich kürzere Vorbereitungszeit für die häufig polymorbiden Patienten, die dann unter Notfallbedingungen operiert werden. Die präoperative Vorbereitung spielt hier also eine entscheidende Rolle und sollte, wann immer möglich, interdisziplinär zusammen mit Geriatern und Internisten erfolgen. Für manche Patienten, die in schlechtem Zustand zur Aufnahme kommen (dekompensierte Herzinsuffizienz, schlecht eingestellter Diabetes, Exsikkose, Anämie oder Elektrolytstörung), kann eine kurze Konditionierungszeit von 12-24 Stunden lebensrettend sein. Die Mehrheit der Patienten profitiert allerdings von der sofortigen Operation. Die Operation stellt eine wirksame Schmerztherapie dar, sie ist die Voraus- setzung für die Mobilisation und Letztere ist die Voraussetzung für die Vermeidung von sekundären Komplikationen wie Dekubitalulzera, Thrombosen, Embolien sowie Pneumonien.

\section{Antikoagulanzien}

Einer der häufigsten Gründe für die verzögerte Versorgung hüftgelenknaher Frakturen ist die Einnahme eines gerinnungshemmenden Präparats. Eine Verzögerung der Operation mit dem Ziel, die Normalisierung der Blutgerinnung abzuwarten, ist jedoch in nahezu allen Fällen unnötig, sodass sich die Auseinandersetzung mit den verschiedenen Substanzgruppen und Wirkungsweisen auch für den Chirurgen lohnt.

\section{Thrombozytenaggregationshemmer}

Sie sollten grundsätzlich weiter gegeben werden. Eine Antagonisierung ist aufgrund der irreversiblen Hemmung der Plättchenfunktion nicht sinnvoll. Das Abwarten der Normalisierung der Plättchenfunktion über mehrere Tage ist aufgrund der bereits erwähnten Zunahme von Mortalität und Komplikationen ebenfalls obsolet und stellt, insbesondere bei auftretender Komplikation, einen klaren Behandlungsfehler dar. $[8,16]$. Ohnehin ist bspw. für Clopidogrel nachgewiesen, dass die Einnahme keinen negativen Einfluss auf das Outcome bei hüftnaher Fraktur hat [19]. Bei auffallend hoher Blutungsneigung intraoperativ kann mit Desmopressin gegengesteuert 
werden. Sonst bleibt die Gabe von Thrombozytenkonzentraten.

\section{Cumarine}

Ihre Auswirkung auf die Blutgerinnung kann verlässlich mit der Bestimmung des INR-Wertes beurteilt werden. In aller Regel kann die Gerinnung mit der i.v. Gabe von Vitamin K innerhalb von 1224 Stunden so weit optimiert werden, dass der Eingriff in gewohnter Weise erfolgen kann. Reicht dies nicht aus oder ist eine sofortige Versorgung angezeigt, kann die Blutgerinnung zielgenau über die Gabe von PPSB (Prothrombin, Prokonvertin, Stuat-Prower-Faktor, AntiHämophiler Faktor B) angehoben werden [20].

Belastbare Daten zeigen, dass chirurgische Eingriffe mit niedrigem Blutungsrisiko unter einer Therapie mit VitaminK-Antagonisten (VKA) durchgeführt werden können, wohingegen bei Eingriffen mit hohem Blutungsrisiko eine Therapie mit Cumarin/Warfarin pausiert werden sollte. Ein Bridging mit Heparin im Falle einer Behandlung mit VKA ist nur noch bei wenigen Patienten mit sehr hohem Thromboembolierisiko indiziert.

\section{Neue direkte orale Antikoagulanzien (NOAK)}

Wegen des sofortigen Wirkungseintritts und der kurzen Halbwertszeit von NOAK erübrigt sich in den meisten Fällen ein Bridging mit Heparinen [21]. Stattdessen kann die Einnahme von NOAK vor dem Eingriff prinzipiell einfach pausiert werden und wieder eingesetzt werden, wenn das Blutungsrisiko vertretbar scheint (i.d.R. 24-48 h postoperativ). Grundsätzlich ist bei Osteosynthesen oder endoprothetischer Versorgung hüftnaher Frakturen eine Mindesttherapiepause von 24 Stunden zu empfehlen. Problematisch ist, dass die zugelassenen NOAK überwiegend renal eliminiert werden und daher bei Niereninsuffizienz kumulieren können. Bei Patienten mit eingeschränkter Nierenfunktion sind NOAK daher, abhängig von der KreatininClearance, u.U. auch für einen längeren Zeitraum abzusetzen [22]. Grundsätzlich fehlen Langzeitdaten und klinische Studien für das perioperative Management zum Umgang mit NOAK bei unfallchirurgischen Eingriffen. Die aktuellen Empfehlungen beruhen im Wesentlichen auf Expertenmeinungen und machen im klinischen Alltag die interdisziplinäre Vorbereitung zwingend erforderlich.

\section{Präoperative Planung}

Die Leitlinien in Orthopädie und Unfallchirurgie gehen von einer Planung der Endoprothesenimplantation in irgendeiner Form, meistens mittels maßstabsgerechter Schablonen oder entsprechender kalibrierter Software aus. Anhand der Übertragung der anatomischen Verhältnisse der gesunden Seite durch Spiegelung auf die kranke Seite kann ein anatomischer Sitz von Pfanne und Schaft und die Größe der Implantate eingeschätzt werden. Für die Diagnostik ist deshalb eine Beckenübersichtsaufnahme mit Kalibrierungs-Tool notwendig.

IT-unterstützte Planungssoftware ermöglicht heute die Planung unter Berücksichtigung von nahezu fast allen Prothesenmodellen. Die Planungen können aus verschiedenen Gründen nicht immer $100 \%$ ig operativ umgesetzt werden. Trotzdem ist es für den Operateur eine gute Vorbereitung, sich mit den Gegebenheiten und den entstehenden Veränderungen durch die Prothesen auseinanderzusetzen und möglichst nahe ans Ziel heranzukommen (siehe Abb. 1, 4 und 5 ).

Besondere Auflagen werden im Deutschen Endoprothesenregister bereits bei der Primärimplantation bei Koxarthrose gefordert. Hier ist aktuell die Implementierung eines Traumamoduls durch die DGU (Deutsche Gesellschaft für Unfallchirurgie) und DGOOC (Deutsche Gesellschaft für Orthopädie und Orthopädische Chirurgie) in Vorbereitung [16].

\section{Zugang und Lagerung}

Für die Endoprothesenimplantation an der Hüfte stehen zahlreiche Zugänge zur Verfügung. Am häufigsten ist der laterale transmuskuläre Zugang nach Bauer und Harding. Mit ihm können alle Arten von Prothesen sicher eingebracht werden. Für die Hemiprothese ist auch der anterolaterale Zugang nach Watson-Jones geeignet.

Für die Lagerung ist allein entscheidend, dass der Operateur sich sicher sein kann, dass er mit ihr das gewünschte Operationsziel ohne Gefährdung der Asepsis optimal erreichen kann.

Der gewählte Zugang zur Hüfte bestimmt die Lagerung. Der Zugang wiederum wird von verschiedenen Operationsschulen gepflegt, die gute Gründe für das gewählte Vorgehen erarbeitet haben. Der häu- figste Zugang ist der transmuskuläre laterale Zugang nach Bauer und Harding, gefolgt vom anterolateralen Watson-JonesZugang und dem sog. hinteren Zugang nach Moore, jeweils modifiziert in minimalinvasiver Technik möglich. Die Lagerung für den lateralen und anterolateralen Zugang ist die Rückenlage auf einem für die Durchleuchtung zugänglichen Tisch. Der Patient liegt dabei exzentrisch zur Seite der zu operierenden Extremität, sodass die Weichteile geringfügig über die Tischkante hängen (Abb.8a und b). Die Lagerung erfolgt auf einer rutschfesten Unterlage, Röntgenschutzvorkehrungen sind i.d.R. störend. Die Abdeckung erfolgt beweglich, wobei besonders darauf zu achten ist, dass die Einmal-U-Tücher auf trockenem Untergrund weit dorsal angeheftet werden, weil sie sich sonst frühzeitig lösen. Die Abdeckung darf die Operation auf keinen Fall beeinträchtigen, auch nicht die Beweglichkeit des Beines, welche für die Resektion des Schenkelhalses und die Manipulation an der Pfanne notwendig ist.

Intraoperative Durchleuchtung muss den Blick auf Azetabulum und proximales Femur ermöglichen. Navigationssysteme, die für die Hüftendoprothetik eingeplant werden, bedürfen einer speziellen Lagerung des Patienten, die auf das jeweilige System abgestimmt sein muss.

Der dorsale Zugang wird in kontralateraler Seitenlage durchgeführt. Der Patient liegt exzentrisch mit den Weichteilen abschließend an der dem Operateur zugewandten Tischkante (Abb.8c und d). Röntgenkontrollen in der axialen Ebene sind erschwert.

Eine generelle Empfehlung für den einen oder anderen Zugang ist nicht möglich. Vielmehr sollte der Zugang verwendet werden, mit dem der jeweilige Operateur die meiste Erfahrung hat. Bezüglich Outcome und Komplikationen unterscheiden sich die genannten Verfahren nur unwesentlich. Möglicherweise besteht für den posterioren Zugang nach Moore ein gering erhöhtes Luxationsrisiko [7]. In jüngerer Zeit kann zudem ein Trend hin zum ventralen Zugang (SmithPetterson) auch in minimalinvasiver Technik verzeichnet werden. Einige Arbeiten berichten hier über Vorteile hinsichtlich Frühmobilisierung, Schmerzen und Patientenzufriedenheit [7,23]. 

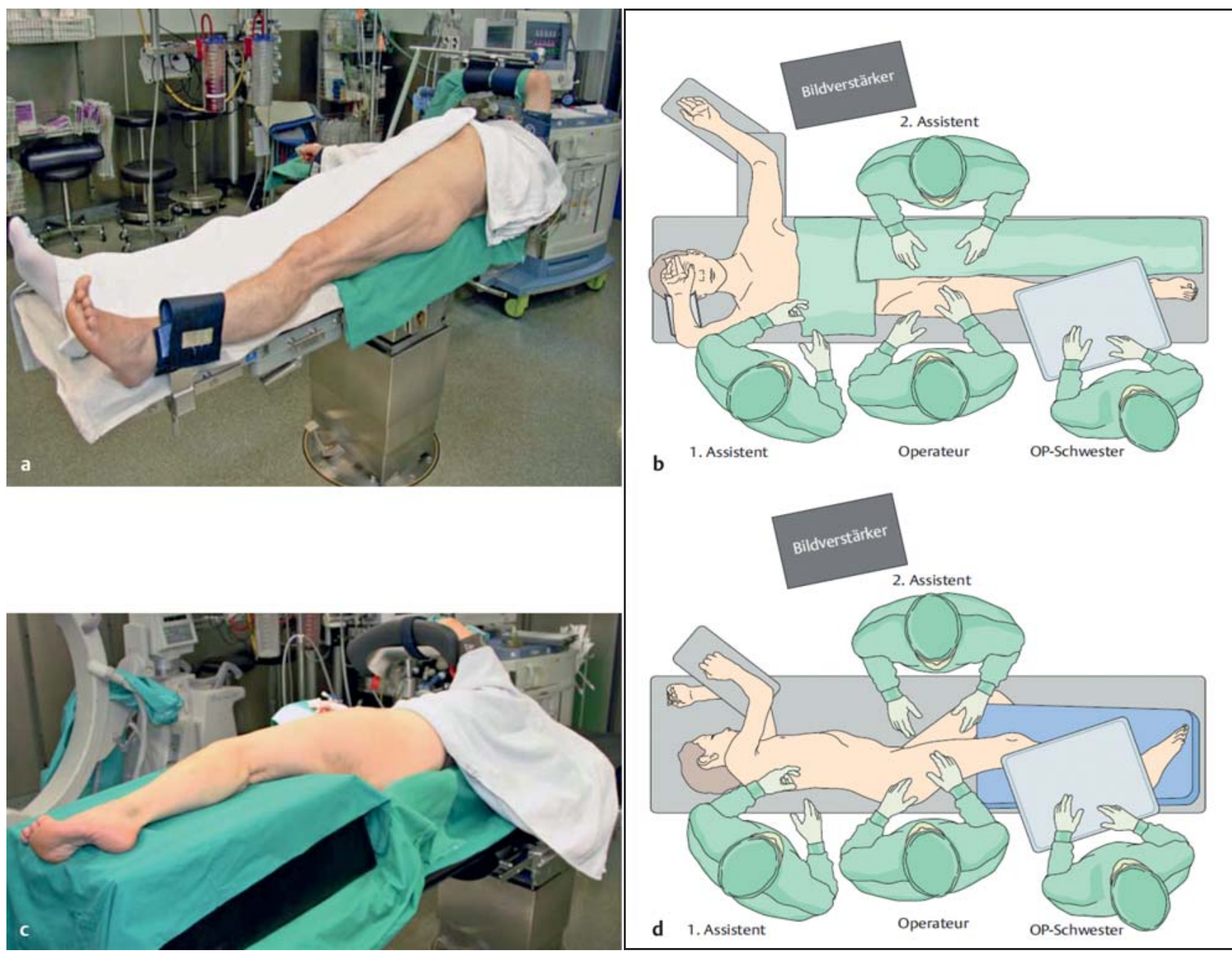

Abb. $\mathbf{8}$ a bis $\mathbf{d}$ a Lagerung des Patienten für anterolateralen und lateralen Zugang. b Anordnung der Personen, die direkt am Patienten bei der Operation arbeiten. Seitenlagerung des Patienten für dorsalen Zugang (c). Anordnung des Operateurs, Assistenten und Operationsschwester (d) (Bild: Bonnaire et al. Planung und Vorbereitung bei Eingriffen im Bereich des proximalen Oberschenkels. OP-Journal 2011; 27: 62-69).

\section{Implantationstechnik}

Wichtig ist bei allen Prothesen die korrekte Implantationstechnik. Vor allem bei Patienten mit kardiopulmonaler Vorbelastung kann es durch Embolisation des Fettmarks vom Femurkanal in die Blutbahn zu Lungenembolien kommen, die tödlich sein können. Als Vorsichtsmaßnahme ist eine gute Abfolge der Maßnahmen sehr hilfreich: Vor der Eröffnung des Markraums sollte mit dem Anästhesisten Kontakt aufgenommen werden und das Risiko des Patienten für eine Embolisation besprochen werden. Dieser wird die Kreislaufsituation stabilisieren, die Oxygenierung und die intravasale Flüssigkeit optimieren. Nach Einverständnis wird der Femurkanal vorsichtig eröffnet und aufgeraspelt. Dabei kann es schon zu einer Druckerhöhung mit Embolisation kommen. Dann erfolgt im Falle der Zementierung eine
Jetlavage, um das Markfett aus der Trabekelmatrix herauszuspülen. Diese Prozedur verbessert das Knochen-ZementInterface und verringert das Risiko für eine fulminante Markfettembolisation. Dann wird der Zementstopper in der korrekten Höhe im Schaft positioniert und der unter Vakuumbedingungen verdichtete Zement retrograd eingebracht. Der Schaft sollte unter kontinuierlichem Anpressdruck zur Aushärtung gebracht werden. Für das Einsetzen der Pfanne ist diese Vorsicht nicht unbedingt geboten, weil es keine Embolisationen von wesentlichem Ausmaß gibt.

Zementfreie Schäfte reduzieren hingegen offenbar das intraoperative Risiko für kardiopulmonale thrombembolische Ereignisse. Die OP-Zeit ist kürzer und der Blutverlust möglicherweise geringer. Die Ergebnisse bez. Reoperationen sind für die jüngeren Patienten mit guter
Knochenqualität ebenfalls vergleichbar $[24,25]$.

Für die Altersgruppe ab 70 besteht klare Evidenz für eine Überlegenheit der Zementierung [3]. Der eingebrachte Zement gewährleistet einen optimalen Formschluss zwischen Knochen und Prothesenschaft und führt zu sofortiger Belastungsstabilität. Insbesondere für die Hemiprothesen konnten deutlich erhöhte intra- und postoperative Komplikationsraten bei der Verwendung zementfreier Schäfte gezeigt werden [26]. Auch bez. der Reoperationsrate für die Totalendoprothesen findet sich im schwedischen Hüftregister kein Vorteil für zementfreie Prothesen bei gleichzeitig erhöhter Reoperationsrate aufgrund intraoperativer Frakturen durch Verwendung zementfreier Schäfte [27]. 
Heutzutage ist eine intra- oder direkt postoperative Röntgendokumentation Pflicht, um Implantationsfehler sofort zu erkennen und zu korrigieren.

\section{Postoperativ}

Die frühe Mobilisation am Folgetag der Operation ist heutzutage mit sofortiger Vollbelastung üblich. Ausnahmen bestätigen die Regel. Eine tägliche Wundkontrolle und eine regelmäßige Kontrolle der Entzündungsparameter (CRP) mit normalerweise kontinuierlichem Abfall derselben lässt Frühinfektionen erkennen. Sonografieuntersuchungen können frühzeitig Hämatome und Flüssigkeitsstraßen erkennen und erleichtern die Entscheidung zur Revision. Im Zweifelsfall sollte man lieber zu früh als zu spät revidieren und Wunde und Gelenk débridieren und spülen. Bei Keimnachweis und 2. Revision wird ein Wechsel der mobilen Komponenten empfohlen. Spätestens nach der 2. Luxation sollte eine Analyse des Luxationsgrunds erfolgen und eine Umsetzung durch Veränderung des Systems veranlasst werden.

\section{Osteoporose}

Zwei Drittel der medialen Schenkelhalsfrakturen, die in Deutschland mit einer Endoprothese versorgt werden, betreffen Frauen um das 80. Lebensjahr, und in nahezu allen Fällen handelt es sich um osteoporoseassoziierte Frakturen [9]. Der überwiegende Anteil dieser Patientengruppe erfüllt damit die Kriterien für eine spezifische Osteoporosetherapie. Diese sollte innerhalb von Netzwerken mit niedergelassenen osteologisch tätigen Ärzten geplant und betreut werden. Zumindest aber die Einleitung einer Basistherapie mit Substitution von Cholecalciferol kann unproblematisch bereits während der stationären Behandlung erfolgen. Eigene Daten zeigen eine fast durchgängig bestehende Mangelversorgung mit Vitamin D speziell in dieser Patientengruppe. Vitamin-D-Spiegel (25OH-Vitamin D) zwischen 30 und $70 \mathrm{ng} /$ $\mathrm{ml}$ führen obendrein zu einer signifikant geringeren Sturzneigung und Frakturrate [28].

\section{Interessenkonflikt: Nein.}

\section{Literatur}

${ }^{1}$ Frerichmann U, Raschke MJ, Stöckle $U$ et al. [Proximal femoral fractures in the elderly. Data from health insurance providers on more than 23 million insured persons-part 2]. Unfallchirurg 2007; 110: 610-616

2 Parker MJ, Gurusamy K. Internal fixation versus arthroplasty for intracapsular proximal femoral fractures in adults. Cochrane Database Syst Rev 2006; CD001708

3 Parker MJ, Gurusamy KS, Azegami S. Arthroplasties (with and without bone cement) for proximal femoral fractures in adults. Cochrane Database Syst Rev 2010; CD001706

${ }^{4}$ Kostuj T, Smektala R, Schulze-Raestrup U et al. The influence of timing of surgery on mortality and early complications in femoral neck fractures, by surgical procedure: an analysis of 22,566 cases from the German External Quality Assurance Program]. Unfallchirurg 2013; 116: 131-137

${ }^{5}$ Moja L, Piatti A, Pecoraro V et al. Timing matters in hip fracture surgery: patients operated within 48 hours have better outcomes. A meta-analysis and meta-regression of over 190, 000 patients. PLoS One 2012; 7: e46175

${ }^{6}$ Simunovic N, Devereaux PJ, Sprague S et al. Effect of early surgery after hip fracture on mortality and complications: systematic review and meta-analysis. CMAJ 2010; 182: 1609-1616

${ }^{7}$ Renken F, Renken S, Paech A et al. Early functional results after hemiarthroplasty for femoral neck fracture: a randomized comparison between a minimal invasive and a conventional approach. BMC Musculoskelet Disord 2012; 13: 141

8 Lein T, Bula P, Jeffries J et al. Fractures of the femoral neck. Acta Chir Orthop Traumatol Cech 2011; 78: 10-19

9 Bonnaire F, Straßberger C, Kieb M et al. [Osteoporotic fractures of the proximal femur. What's new?]. Chirurg 2012; 83: 882-891

${ }^{10}$ Burgers PT, Van Geene AR, Van den Bekerom MPJ et al. Total hip arthroplasty versus hemiarthroplasty for displaced femoral neck fractures in the healthy elderly: a meta-analysis and systematic review of randomized trials. Int Orthop 2012; 36: 1549-1560

${ }^{11}$ Miyamoto RG, Kaplan KM, Levine BR et al. Surgical management of hip fractures: an evidence-based review of the literature. I: femoral neck fractures. J Am Acad Orthop Surg 2008; 16: 596-607

12 Jameson SS, Jensen CD, Elson DW et al. Cemented versus cementless hemiarthroplasty for intracapsular neck of femur fracture - a comparison of 60,848 matched patients using national data. Injury 2013; 44: 730-734

${ }^{13}$ Kenzora JE, Magaziner J, Hudson J et al. Outcome after hemiarthroplasty for femoral neck fractures in the elderly. Clin Orthop Relat Res 1998; 348: 51-58

14 Osarumwense D, Tissingh E, Wartenberg $K$ et al. The Targon FN system for the management of intracapsular neck of femur fractures: minimum 2-year experience and outcome in an independent hospital. Clin Orthop Surg 2015; 7: 22-28

15 Brémant IJ. [10 years follow-up of the M.E. Müller self-locking cemented total hip prosthesis]. Rev Chir Orthop Reparatrice Appar Mot 1995; 81: 380-388
16 Strohm PC, Raschke M, Hoffmann R et al. [Hip fracture prosthetics in German trauma surgery. State of the art]. Unfallchirurg 2015; 118: 173-176

${ }^{17}$ Roche JJ, Wenn RT, Sahota O et al. Effect of comorbidities and postoperative complications on mortality after hip fracture in elderly people: prospective observational cohort study. BMJ 2005; 331: 1374

${ }^{18}$ Leonardsson O, Rogmark C, Kärrholm J et al. Outcome after primary and secondary replacement for subcapital fracture of the hip in 10264 patients. J Bone Joint Surg Br 2009; 91: 595-600

${ }^{19}$ Wordsworth DR, Halsey T, Griffiths R et al. Clopidogrel has no effect on mortality from hip fracture. Injury 2013; 44: 743-746

20 Dettoni F, Peveraro A, Dettoni A et al. Epidemiology of hip fractures in northwestern Italy: a multicentric regional study on incidence of hip fractures and their outcome at 3-year follow-up. Musculoskelet Surg 2012; 96: 41-46

21 Schulman S, Carrier M, Lee AY et al. Perioperative management of dabigatran: a prospective cohort study. Circulation 2015; 132: 167-173

22 Baron TH, Kamath PS, McBane RD. New anticoagulant and antiplatelet agents: a primer for the gastroenterologist. Clin Gastroenterol Hepatol 2014; 12: 187-195

${ }^{23}$ Unger AC, Dirksen B, Renken FG et al. Treatment of femoral neck fracture with a minimal invasive surgical approach for hemiarthroplasty - clinical and radiological results in 180 geriatric patients. Open Orthop J 2014; 8: 225-231

${ }^{24}$ Ning GZ, Li YL, Wu Q et al. Cemented versus uncemented hemiarthroplasty for displaced femoral neck fractures: an updated metaanalysis. Eur J Orthop Surg Traumatol 2014; 24: 7-14

${ }^{25}$ Ahn J, Man LX, Park S et al. Systematic review of cemented and uncemented hemiarthroplasty outcomes for femoral neck fractures. Clin Orthop Relat Res 2008; 466: 2513-2518

${ }^{26}$ Gierer P, Mittlmeier T. [Femoral neck fracture]. Unfallchirurg 2015; 118: 259-269

${ }^{27}$ Hailer NP, Garellick G, Kärrholm J. Uncemented and cemented primary total hip arthroplasty in the Swedish Hip Arthroplasty Register. Acta Orthop 2010; 81: 34-41

28 Qiu S, Rao DS, Palnitkar S et al. Independent and combined contributions of cancellous and cortical bone deficits to vertebral fracture risk in postmenopausal women. J Bone Miner Res 2006; 21: 1791-1796

\section{Prof. Dr. Felix Bonnaire \\ Chefarzt}

Dr. Philipp Bula

Leitender Oberarzt/Stellv. Chefarzt

Klinik für Unfall-, Wiederherstellungsund Handchirurgie

Überregionales Traumazentrum

AO Fellowship Host Center

Städtisches Krankenhaus Dresden-

Friedrichstadt

Friedrichstraße 41

01067 Dresden

bonnaire-fe@khdf.de 